\title{
PICOSECOND SELF-MODULATION OF THE FUNDAMENTAL ABSORPTION OF A LIGHT PULSE IN THE GaAs, THAT GENERATES THE STIMULATED EMISSION, CAUSING INTERBAND ELECTRON OSCILLATIONS
}

\author{
N. N. Ageeva, I. L. Bronevoi, A. N. Krivonosov, D.N. Zabegaev \\ Kotelnikov Institute of Radioengineering and Electronics of Russian Academy of Sciences, \\ Mokhovaya 11-7, Moscow 125009, Russia
}

The paper is received on March 25, 2019

\begin{abstract}
A brief review of experimental results is presented on discovery of a new nonlinear phenomenon - picosecond self-modulation of the fundamental absorption of a picosecond probe light pulse in a thin layer of GaAs that generates stimulated intense picosecond emission. It is explained by the synchronization of two modulations of the electron population of energy levels in the conduction band. One is created by the probing light. The other is created at the bottom of the zone by picosecond interband oscillations of electrons in the emission field and is transmitted up the zone to restore the detailed equilibrium of electron transitions with the radiation and absorption of LO phonons. Synchronization of modulations occurs in order to achieve similar detailed equilibrium.
\end{abstract}

Key words: picosecond, self-modulation, fundamental light absorption, stimulated emission, synchronization, interband electron oscillations, GaAs.

In a cycle of interrelated, mainly experimental works, a new nonlinear phenomenon was discovered - picosecond self-modulation of the fundamental absorption of a picosecond probing light pulse in a thin $(\sim 1 \mu \mathrm{m}) \mathrm{GaAs}$ layer. The latter is an active resonator in the composition of the $\mathrm{Al}_{\mathrm{x}} \mathrm{Ga}_{1-\mathrm{x}} \mathrm{As}-\mathrm{GaAs}-\mathrm{Al}_{\mathrm{x}} \mathrm{Ga}_{1-\mathrm{x}} \mathrm{As}$ heterostructure (with antireflection coating) and is subjected to powerful picosecond optical pumping. During pumping, stimulated (amplified spontaneous) intense (up to $1 \mathrm{GW} / \mathrm{cm}^{2}$ ) picosecond emission occurs in the layer. The absorption of the spectral component (width $\delta \hbar \omega_{p} \approx 0.5 \mathrm{meV}$ ) of the probe pulse from the region of the maximum of its spectrum was measured. Pulse duration at half-height (FWHM) was 
about 10 ps. The scheme of optical transitions in such an experiment is shown in Fig.1.

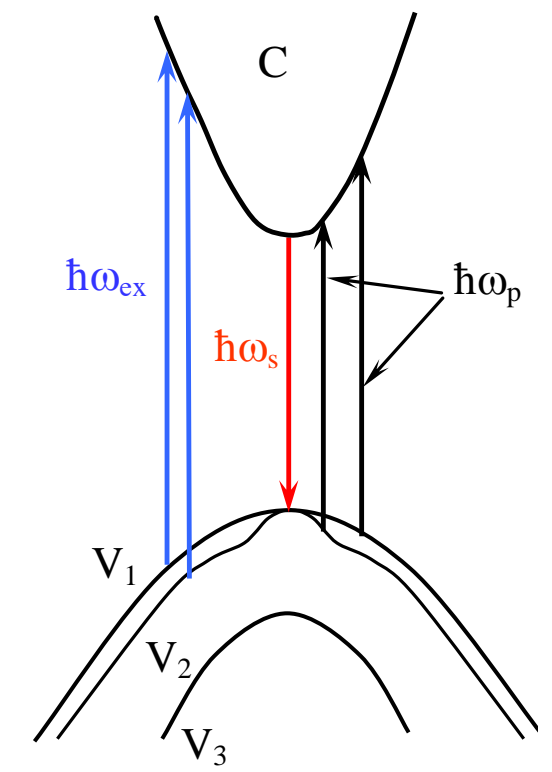

Fig. 1. Scheme of optical transitions. $\mathrm{V}_{1}, \mathrm{~V}_{2}$ are the valence bands of heavy and light holes, $\mathrm{V}_{3}$ is the spin-orbitally split zone, $\mathrm{C}$ is the conduction band.

Possible electron transitions are shown for pumping, probing, and stimulated emission with photon energies $\hbar \omega_{e x}, \hbar \omega_{p}$, and $\hbar \omega_{s}$, respectively

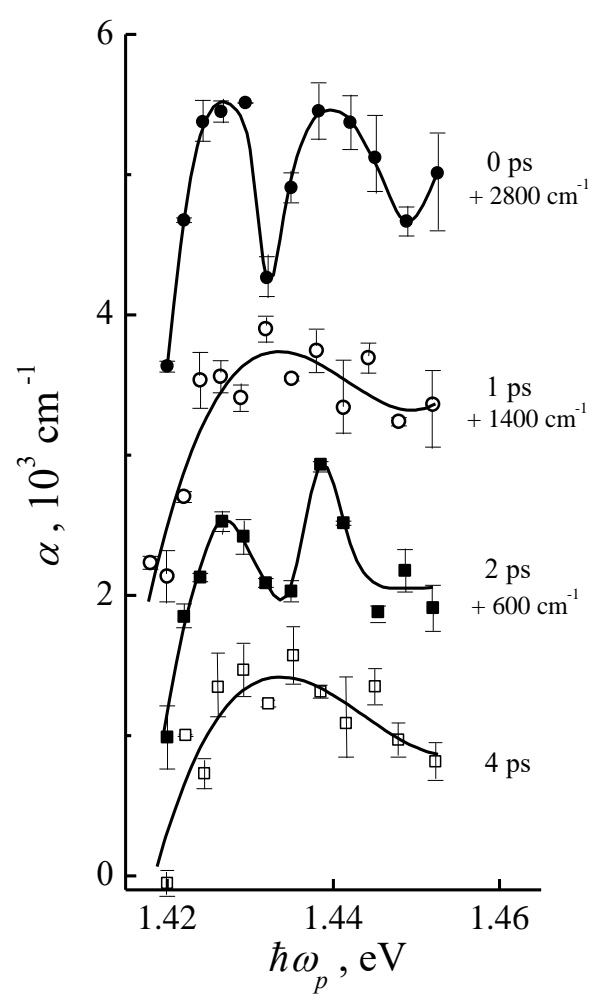

Fig. 2. Absorption spectra $\alpha\left(\hbar \omega_{p}\right)$ measured at fixed $W_{e x}$ and $\hbar \omega_{e x}$ and different $\tau$ which is indicated to the right of the curves. The spectra are shifted along the ordinate axis by the value indicated at the right

The change in the absorption coefficient $\alpha$ of the total energy of the indicated component was measured by the correlation method, and the instantaneous value of the absorption coefficient $\alpha_{M}$ was measured in real time $t$. The following dependences of $\alpha$ turned out to be modulated: 1) on the photon energy of the probe pulse $\hbar \omega_{p}$, Fig. 2 and others, [1-3];2) on the time delay $\tau$ of this pulse relative to the pumping, Fig.3, [4,5]; 3) on pump pulse energy $W_{e x}$, Fig.4 (plots $\alpha\left(W_{e x}\right)$ differ in agreement with changes of the intensity of emission $I_{s}$ in space in the active region), [4]. The modulation of the absorption spectrum $\alpha\left(\hbar \omega_{p}\right)$ oscillated with a change in $\tau$, Fig.2 [1,3] and $W_{e x}$, Fig.5, [3,6] and was still amplitude-modulated with a period 
$\Delta=\hbar \omega_{L O}\left(1+m_{e} / m_{h}\right)$, Fig.6, [2], where $\hbar \omega_{L O}$ is the energy of a longitudinal optical (LO) phonon, $m_{e}$ and $m_{h}$ are the electron and heavy hole masses, respectively.

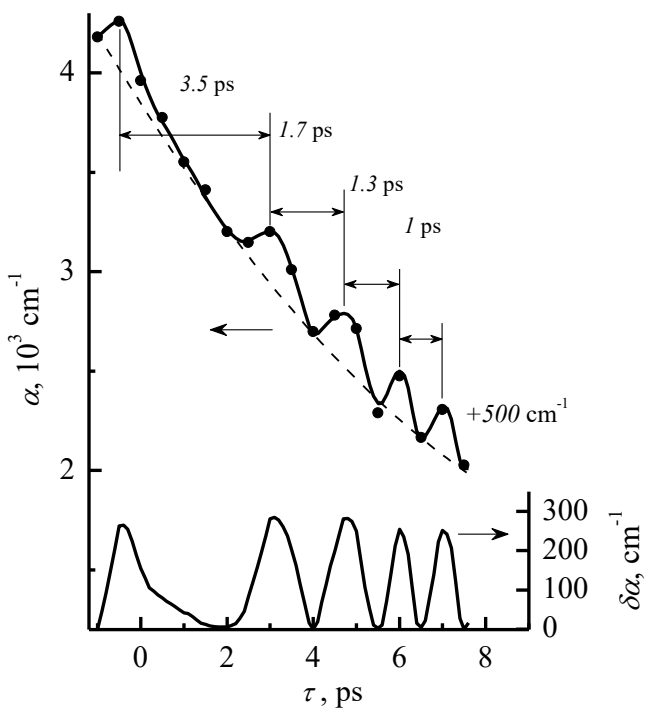

Fig. 3. Dependence $\alpha(\tau)$ at fixed $W_{e x}$, $W_{p}$ and $\hbar \omega_{p}$. Dashed line is a smoothly decreasing component $\alpha_{1}(\tau)$ of the dependence $\alpha(\tau)$. Oscillation component $\delta \alpha(\tau)=\alpha(\tau)-\alpha_{1}(\tau)$

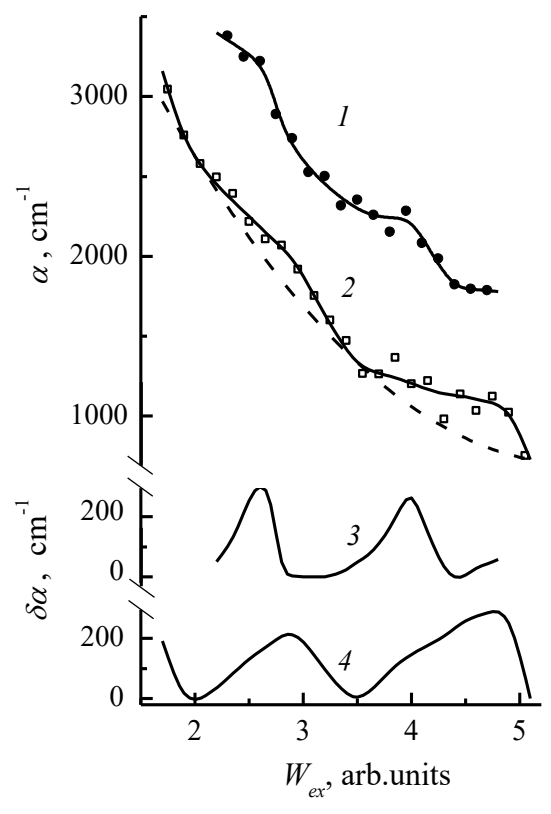

Fig. 4. 1 - Dependence of $\alpha\left(W_{e x}\right)$ at fixed $\hbar \omega_{p}, \tau$ and $W_{p}$ and various values of $\theta$, $\mu \mathrm{m}: 0-2 ; 77-1 . \theta$ is the distance between the centers of the focal spots of the pump and probe beams on the sample.

The dotted line shows the smoothly decreasing component $\alpha_{2}\left(W_{e x}\right)$ of the dependence $\alpha\left(W_{e x}\right)$. Oscillating component $\delta \alpha\left(W_{e x}\right)=\alpha\left(W_{e x}\right)-\alpha_{2}\left(W_{e x}\right)$ at $\theta$, $\mu \mathrm{m}: 0$ - $4 ; 77-3$

The modulation depended on the ratio of the probing pulse energy $W_{p}$ and pumping $W_{e x}$ up to disappearance and again formation, Fig.7, [5]. The inverse period $T^{-1}$ of the spectrum modulation oscillations that occurred with a change of $\tau$ increased with an increase of $W_{e x}$, and in fact, due to the increase of $I_{s}$ caused by the latter, Fig.8, $[3,7,8]$. Phase modulation characteristic at maximum $W_{e x}$ had the shape of a meander with the phase jump of $\pi$, Fig.9, [9] and was determined by stimulated Raman scattering (SRS) of the spectral emission modes that were eigenmodes of the GaAs resonator, Fig.10, [10]. And the main experimental evidence: picosecond modulation 
of dependence $\alpha_{M}(t)$ was registered, Fig.11. Its period (varying in the range 6-13 ps with variation of $\tau$ ) and coefficient $\alpha$, both considered as functions of $\tau$, turned out to be anti-correlated, Fig.12, [5].

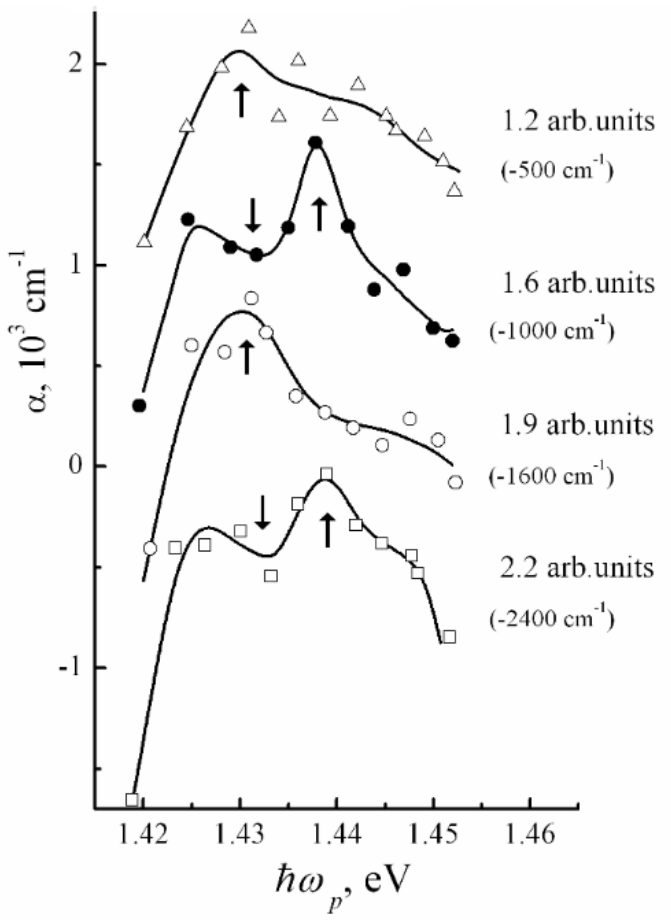

Fig. 5. The absorption spectra $\alpha\left(\hbar \omega_{p}\right)$, measured at the delay $\tau=3$ ps and different pump energies $W_{e x}$, indicated to the right of the curves. The spectra are shifted along the ordinate axis by the value indicated in brackets

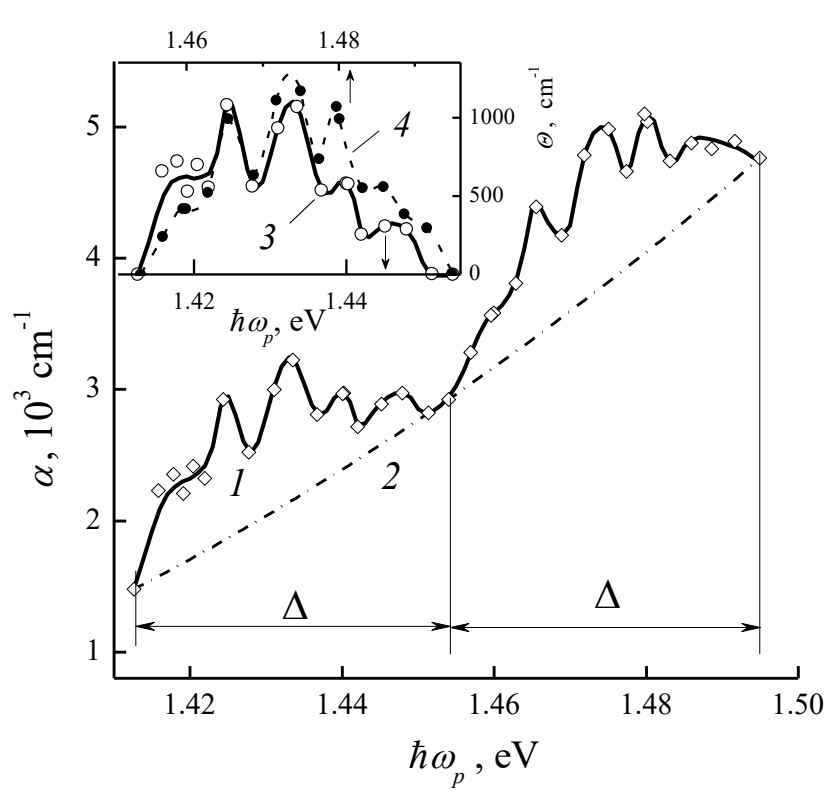

Fig. 6. 1 - light absorption spectrum, measured at $\tau=-0.3 \mathrm{ps}$ and fixed energy $W_{e x} ; 2$ - a parabola segment tangent to the spectrum 1 . Inset: the difference $\Theta$ between the absorption spectrum 1 and the tangent 2 in the spectral intervals $\hbar \omega, \mathrm{eV}: 3-1.413-$ $1.454 ; 4-1.454-1.495 \mathrm{eV}$

The modulation manifestations listed above are explained (partly quantitatively, otherwise qualitatively) by the following processes that are found in the course of the research. Under the influence of intrinsic intense stimulated emission, the following phenomena take place in the GaAs conduction band: 1) the slowing down of elimination of deviations from Fermi distribution of charge carriers which is caused by their energy transport and the Pauli prohibition (but the electron transitions indicated by 2 in Fig.10 are intensified by light exposure when they become an integral part of SRS, as with active laser spectroscopy) [10]; 2) depletion 
of the population of energy levels from which electrons recombine, fig.13, [11,12]; 3) modulation of the depleted population by interband electron oscillations excited by the emission field [7] and synchronized SRS [10] (the estimate of the frequency of oscillations using perturbation theory is close to $T^{1}$, Fig. 8, $\left.{ }^{*}[7,8]\right)$; 4) translation (with period $\hbar \omega_{L O}$ ) of population depletion and its modulation up the zone to restore the detailed equilibrium of electron transitions with radiation and with absorption of LO-phonons (the distribution of electrons from the bottom of the zone to the level to which they are pumped becomes modulated and deviates from the Fermian one) [10]; 5) synchronization of two optically excited modulations of the population of the levels of the conduction band (occurring again for the specified detailed equilibrium), in particular, synchronization of the above-described modulation with the modulation created by the probe pulse, [5].

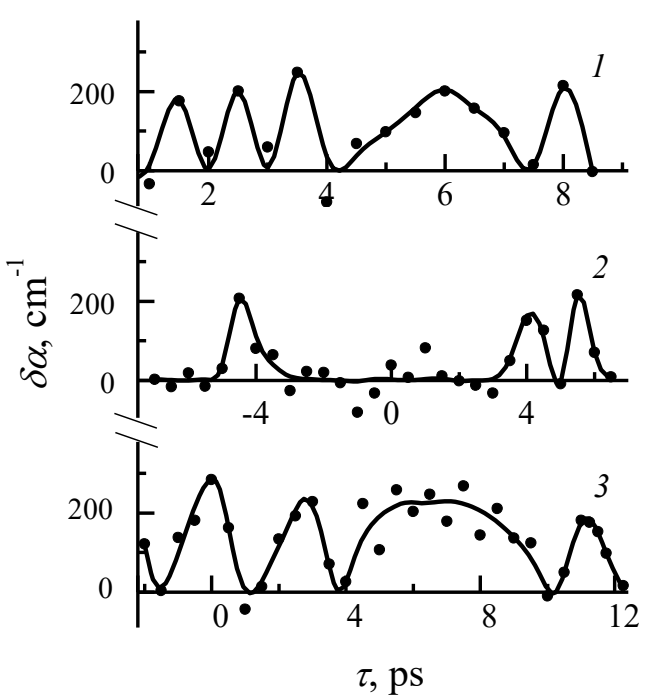

Fig. 7. $\delta \alpha(\tau)$ is the oscillatory component of the dependence $\alpha(\tau)$ : $W_{e x}=2.5$ arb. units and $W_{p}=12$ arb. units - (1); $W_{e x}=3.5$ arb. units and $W_{p}$ $=22$ arb. units $-(2), W_{e x}=3.5$ arb. units and $W_{p}=27$ arb. units $-(3)$; Solid lines are drawn for clarity

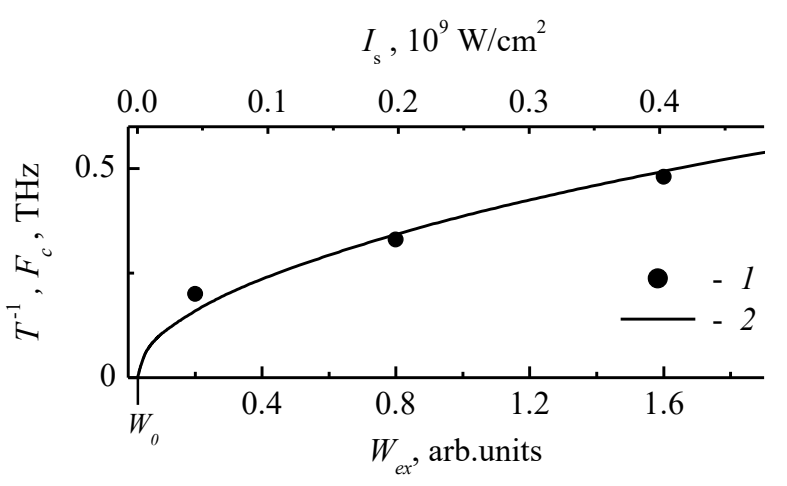

Fig. 8. The inverse period $T^{1}$ of the oscillations of the modulation of the absorption spectrum with varying $\tau$, presented as a function of $W_{e x}-(1) . W_{0}$ is the pump energy threshold for the stimulated emission. Estimated dependence of the frequency of interband electron oscillations $F_{c}$ on the emission intensity $I_{s}-(2)$

Experimental points shown in Fig.8, for each pump pulse were measured in the region of pulse maximum, where the pumping is close to quasi-stationary. The dependence $T^{-1}\left(I_{s}\right)$ is steeper, if it is measured on the front and on the decay of the pump pulse (and emission). That can be caused by the following fact. In these latter cases, the frequency- (and therefore phase-) modulated population of energy levels by charge carriers is probed. 


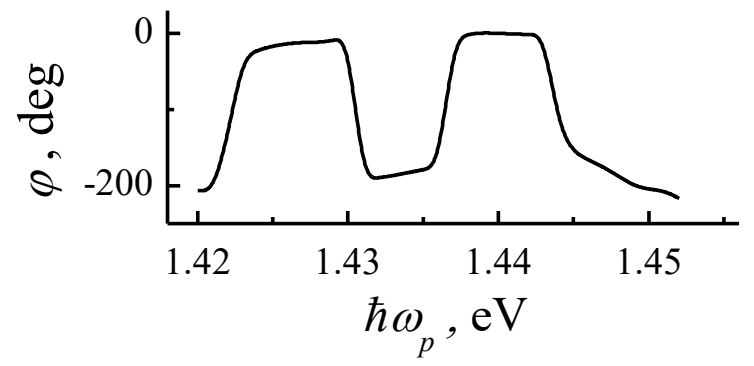

Fig. 9. Phase constant of the modulation of the absorption of the probe pulse as a function of its photon energy

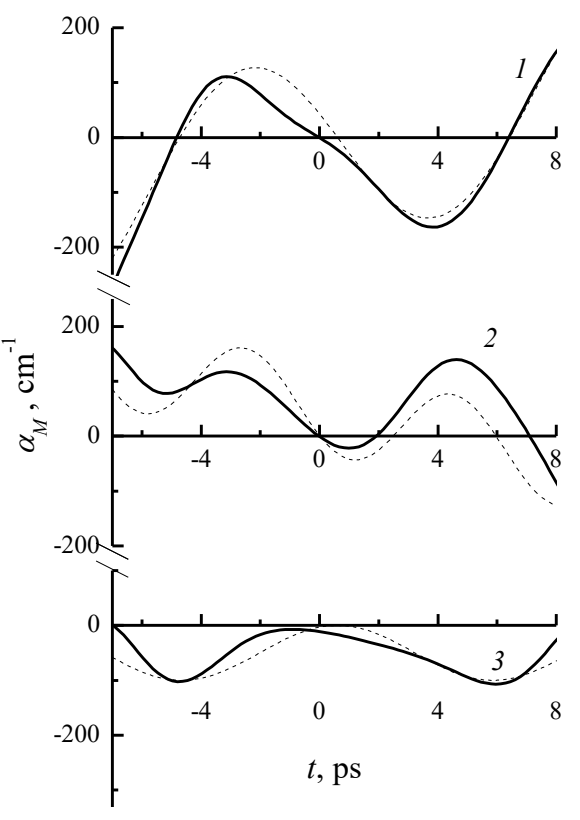

Fig. 11. Dependence $\alpha_{M}(t)$ (solid lines) and its sinusoidal approximation (dotted line) for $\tau$, ps: $1-(-1), 2-0$,

$$
3-2
$$

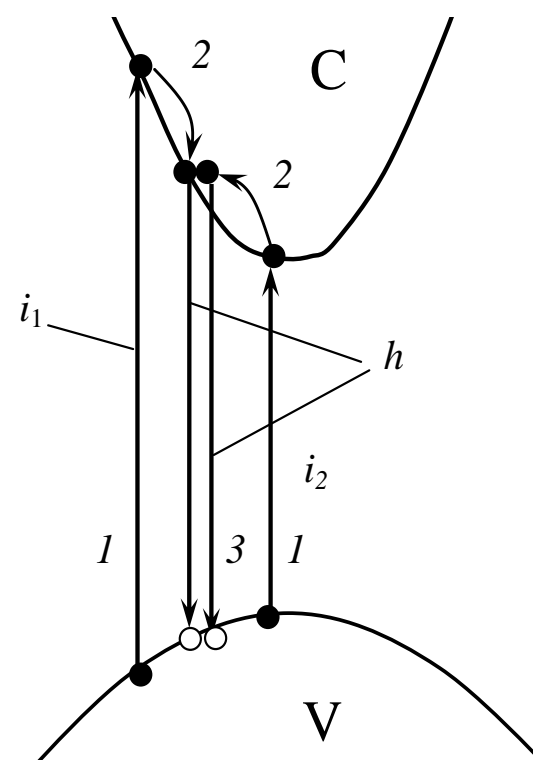

Fig.10. Scheme of stimulated Raman scattering of spectral $i_{1^{-}}, i_{2^{-}}$and $h$ emission modes

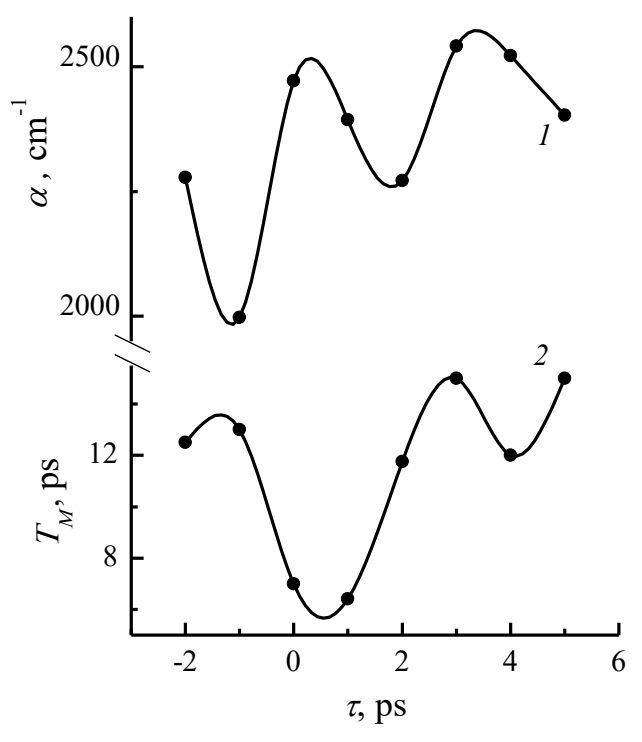

Fig. 12. Dependence on the delay $\tau$ of the absorption coefficient $\alpha-(1)$ and the $T_{M}$ period of the picosecond modulation of dependence $\alpha_{M}(t)-(2)$ 


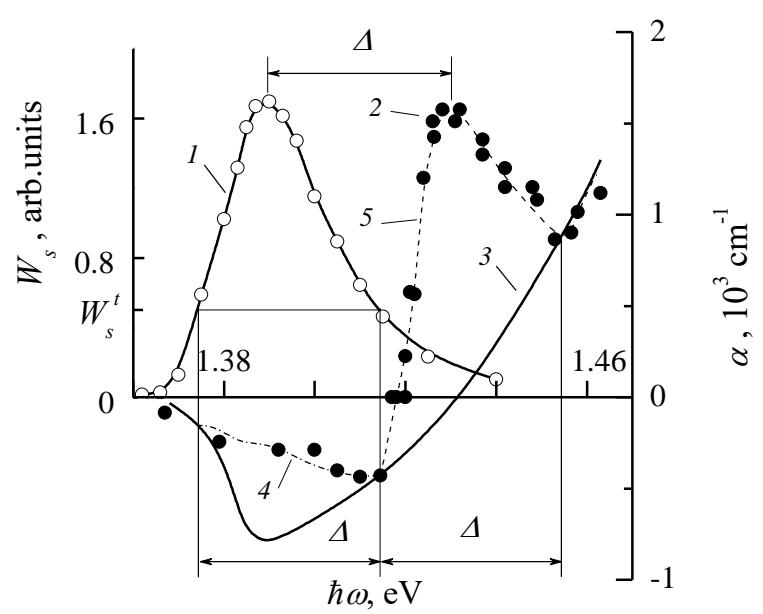

Fig. 13. The GaAs emission spectrum

$W_{s}(\hbar \omega)-1$. The light absorption spectrum for GaAs $\alpha(\hbar \omega)$ at $\tau=-3$ ps (similar to the spectra in Fig. 2 at $\tau=$ 1 and $4 \mathrm{ps}$ ): -2 . The absorption spectrum, calculated for the EHP Fermi distribution $\alpha_{F D}(\hbar \omega)-(3)$ at $T_{c}$ $=52 \mathrm{meV}, n=p \approx 4.7 \times 10^{18} \mathrm{~cm}^{-3} .4-$ $\alpha_{F D}(\hbar \omega)+b\left\{W_{s}(\hbar \omega)-W_{s}^{t}\right\} ; 5-$ $\alpha_{F D}(\hbar \omega)+b_{1}\left\{W_{s}\left(\hbar \omega-\hbar \omega_{L O}\right)-W_{s}^{t}\right\} . \hbar \omega_{e x}=$ $1.558 \mathrm{eV}$

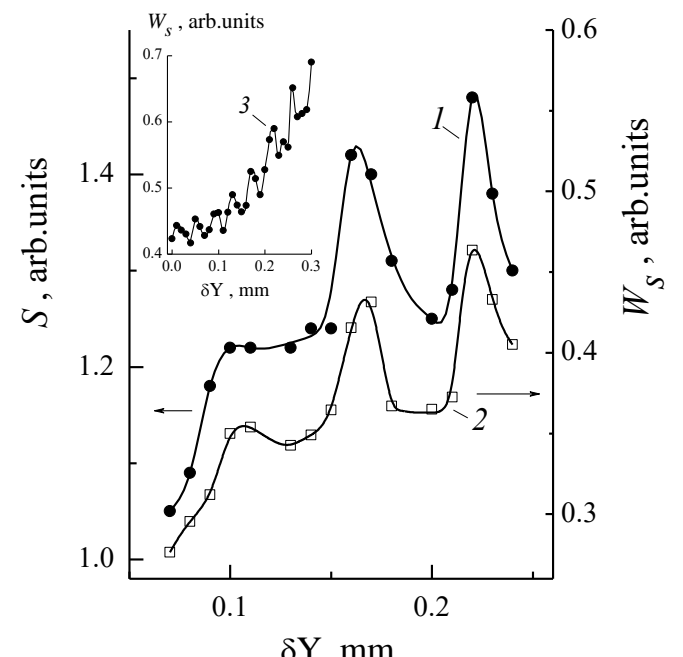

Fig. 14. Dependences on the shift $\delta Y$ of the focal pumping spot to the end of the sample: 1 - emission energy $\mathrm{S}$ from the end face integrated over the spectrum (in the range $\hbar \omega_{s}=1.3852 \mathrm{eV} \div 1.3898$ $\mathrm{eV}) ; 2$ and 3 (inset) - the emission energy $W_{s}$ at a photon energy $\hbar \omega_{s}=$ $1.3858 \mathrm{eV}$ (curve 3 was measured in a separate experiment, where the emission energy was higher than in the case when curve 2 was measured)

This synchronization is similar to synchronization of coupled oscillators. A significant initial desynchronization of the latter can lead to the "quenching" of oscillations, similar to the disappearance of modulation in some $W_{e x} / W_{p}$ relations. During interband oscillations, electrons emit and absorb photons of emission. The emission modulation, arising from this, was confirmed by: (a) the modulation of the characteristics of the emission, explained by the modulation of the emission with 4 ps period, decreasing with increasing $W_{e x}$, Fig.14-16, [13]; (b) modulation of an intense $\left(1 \mathrm{GW} / \mathrm{cm}^{2}\right)$ picosecond light pulse of a semiconductor laser, found by Vasiliev with the autocorrelation method [14].

Let us denote by index " $p$ " the level of the conduction band to which electrons transfer from the valence band upon absorption of the probe pulse. The absorption of this pulse depends, in particular, on the population of the p-level. The flow of the electrons (emitting LO-phonons) from the p-level to the level from which they are stimulated to recombine depends on the desynchronization of the modulations created 
by the emission and the probe pulse.

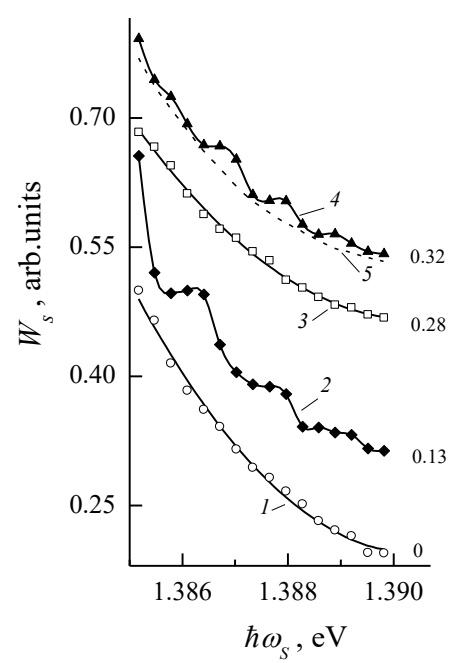

Fig. 15. Emission spectra at different shifts: $1-\delta Y=0.16 \mathrm{~mm} ; 2-\delta Y=$ $0.18 \mathrm{~mm} ; 3-\delta Y=0.20 \mathrm{~mm} ; 4-\delta Y=$ $0.24 \mathrm{~mm} .5$ - the parabola section tangent to the spectrum. For clarity, each curve is shifted along the ordinate axis relative to the true position by the amount indicated to the right of the curve.

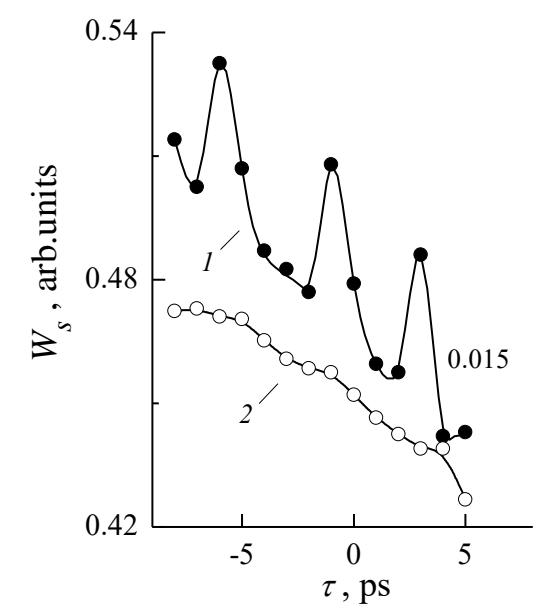

Fig. 16. Dependence on the delay $\tau$ energy $W_{s}$ of emission with a photon energy: $1-\hbar \omega_{s}=1.3867 \mathrm{eV}, 2-\hbar \omega_{s}=$ $1.3877 \mathrm{eV}, \delta Y=0.15 \mathrm{~mm}$, with powerful pump and probe pulses. Curve 1 is shifted along the ordinate axis relative to the true position by the amount indicated to the right of the curve.

The desynchronization was changed by variation of: the delay $\tau$, the frequency of interband oscillations (changing $I_{s}$ ), the "probed" energy level of the conduction band (changing $\hbar \omega_{p}$ ). With such a change in desynchronization, in accordance with the change in the time-integrated probing of the above-stated electron flow, the absorption coefficient $\alpha$ will also change (and the energy of the corresponding spectral component of the emission, which was also observed, Fig. 16, [5,13]). The change the non-integrated electron flow in real time is accompanied by a corresponding change in the instantaneous absorption of the probing light and is depicted by the modulation of the $\alpha_{M}(t)$ dependence. These two factors directly lead to the above-stated experimentally detected manifestations of modulation, necessary for its explanation.

The authors are deeply grateful to Yu.V. Andreev for discussing the manuscript of the article.

The work was carried out within the framework of the state task. 


\section{References}

1. Ageeva N.N., Bronevoi I.L., Krivonosov A.N., Stegantsov S.V. Ultrafast SelfModulation of the Optical Absorption Spectrum Under Conditions of Both the Ultrashort Optical Pumping and Superluminescence in GaAs. Semiconductors. 2006. Vol.40, No.7. P.785-793.

2. Ageeva N.N., Bronevoi I.L., Krivonosov A.N., Nalet T.A., Stegantsov S.V. Participation of Electron-Phonon Interaction in the Ultrafast Self-Modulation of Absorption of Light in GaAs. Relation of Modulation of Absorption with the Spectrum of Stimulated Radiation in GaAs, Semiconductors. 2007. Vol.41. No.12. P.1398-1404.

3. Ageeva N.N., Bronevoi I.L., Krivonosov A.N., Nalet T.A. Cyclic Behavior of Ultrafast Self-Modulation of the Light-Absorption Spectrum under Conditions of Pump and Stimulated Emission in GaA. Semiconductors. 2008. Vol.42, No.9. P.1037-1043.

4. Ageeva N.N., Bronevoi I.L., Zabegaev D.N., Krivonosov A.N. Oscillations of Absorption of a Probe Picosecond Light Pulse Caused by Its Interaction with Stimulated Picosecond Emission of GaAs. JETP. 2015. Vol.120, No.4. P.664671.

5. Ageeva N.N., Bronevoi I.L., Zabegaev D.N., Krivonosov A.N. SelfSynchronization of the Modulation of Energy-Levels Population with Electrons in GaAs Induced by Picosecond Pulses of Probe Radiation and Intrinsic Stimulated Emission. Semiconductors. 2016. Vol.50, No.10. P.1312-1321.

6. Ageeva N.N., Bronevoi I.L., Zabegaev D.N., Krivonosov A.N. Repetition of the Shape of the Ultrafast Self-Modulation of the Optical Absorption Spectrum upon Varying the Energy of Pulse of GaAs Pumping. Semiconductors. 2010. Vol.44, No.10. P.1285-1288.

7. Ageeva N.N., Bronevoi I.L., Zabegaev D.N., Krivonosov A.N. Subterahertz Self-Oscillations of Depletion of Electron Populations in the Conduction Band of GaAs in the Presence of Pumping and Intrinsic Stimulated Radiation. Semiconductors. 2010. V.44, No.9. P.1121-1128. 
8. Ageeva N.N., Bronevoi I.L., Zabegaev D.N., Krivonosov A.N. Modulation of characteristics of picosecond stimulated emission of GaAs, recommendations about its use and suppression. Visualization of the hole, "burnt" by emission, in the region of amplification of light absorption spectrum. Zhurnal Radioelektroniki - Journal of Radio Electronics. 2012. No. 10. Available at http://jre.cplire.ru/jre/oct12/3/text.html. (In Russian)

9. Ageeva N.N., Bronevoi I.L., Krivonosov A.N. Subterahertz Self-Oscillations in Ultrafast Self-Modulation of Optical Absorption in GaAs. Semiconductors. 2008. Vol.42. No.12. P.1395-1402.

10. Ageeva N.N., Bronevoi I.L., Zabegaev D.N., Krivonosov A.N. Switching of Spectral Modes of Picosecond Stimulated Radiation of GaAs due to Stimulated Raman Scattering in the Presence of Interband Oscillations of Electrons in the Radiation Field. Journal of Communications Technology and Electronics. 2018. No.63(10). P.1235-1244.

11. Bronevoi I.L., Krivonosov A.N., Perel' V.I. Phonon Oscillations in the Spectrum of the Reversible Bleaching of Gallium Arsenide under Interband Absorption of a High-Power Picosecond Light Pulse. Solid State Communications. 1995. Vol.94, No. 9. P.805-808.

12. Ageeva N.N., Bronevoi I.L., Krivonosov A. N., Kumekov S.E., Stegantsov S.V. "LO-Phonon" Correlation between Picosecond Superluminescence Spectrum and Special Features of Absorption Spectrum in GaAs for Non-Fermi Distribution of Carriers Induced by Picosecond Light Pulse. Semiconductors. 2002. Vol.36, No.2. P.136-140.

13. Ageeva N.N., Bronevoi I.L., Krivonosov A. N., Kumekov S.E., Nalet T.A., Stegantsov S.V. Modulation of the Characteristics of Intense Picosecond Stimulated Emission from GaAs. Semiconductors. 2005. Vol.39. No.6. P.650657.

14. Vasil'ev P.P. Experimental observation of coherent optical oscillations in picosecond semiconductor lasers under strong-field conditions. Quantum Electronics. 1994. Vol.24. No.6. P.540-542. 


\section{For citation:}

N. N. Ageeva, I. L. Bronevoi, A. N. Krivonosov, D.N. Zabegaev. Picosecond self-modulation of the fundamental absorption of a light pulse in the GaAs, that generates the stimulated emission, causing interband electron oscillations. Zhurnal Radioelektroniki - Journal of Radio Electronics. 2019. No.4. Available at http//jre.cplire.ru/jre/apr19/2/text.pdf

DOI $10.30898 / 1684-1719.2019 .4 .2$ 